\title{
Fern Constituents: Triterpenoids from Adiantum capillus-veneris
}

\author{
Takahisa NAKAne, ${ }^{a}$ Yoshiko MAedA,${ }^{a}$ Hideharu Ebihara,${ }^{a}$ Yoko AraI,${ }^{a}$ Kazuo Masuda,${ }^{a}$ \\ Akihito Takano, ${ }^{a}$ Hiroyuki Ageta, ${ }^{a}$ Kenji Shionima, ${ }^{* a}$ Shao-Qing $\mathrm{CAI}^{,},{ }^{b}$ and \\ Osama Bashir ABDEL-HALIM ${ }^{c}$ \\ ${ }^{a}$ Showa Pharmaceutical University; Machida, Tokyo 194-8543, Japan: ${ }^{b}$ Division of Pharmacognosy, School of \\ Pharmaceutical Sciences, Peking University; 38 Xue-yuan Road, Haidian District, Beijing 100083, China: and ${ }^{c}$ Mansoura \\ University; 35511 Mansoura, Egypt. Received April 18, 2002; accepted May 31, 2002
}

\begin{abstract}
Two new migrated hopane triterpenoids, viz. $4 \alpha$-hydroxyfilican-3-one and fern-9(11)-en-12 $\beta$-ol, and olean18-en-3-one and olean-12-en-3-one as the first example of oleanane compounds from Adiantum ferns were isolated along with many other known triterpenoids from Adiantum capillus-veneris of China and Egypt. Their structures were elucidated by spectroscopic analyses.
\end{abstract}

Key words Adiantum capillus-veneris; triterpenoid; Adiantaceae; $4 \alpha$-hydroxyfilican-3-one; fern- $9(11)$-en-12 $\beta$-ol

In preceding papers of this series, ${ }^{1,2)}$ we reported the isolation of twenty-two triterpenoids, including nine new compounds from the fresh fronds of Adiantum capillus-veneris L. (Adiantaceae) collected in Japan. Further investigation of $A$. capillus-veneris L. collected in China and Egypt has resulted in the isolation of two new triterpenoids; viz. $4 \alpha$-hydroxyfilican-3-one (1) and fern-9(11)-en-12 $\beta$-ol (2) of Chinese origin, and two oleanane triterpenoids; olean-12-en-3-one (3) and olean-18-en-3-one (4) of Egyptian origin (Chart 1). In fact, 3 and $\mathbf{4}$ are the first examples of oleanane compounds from Adiantum ferns. This paper deals with the isolation and structure elucidation of these new compounds.

The constituents of the crude hexane extract of these fronds were purified by various chromatographic techniques (see Experimental) to give nineteen triterpenoids, seventeen of which were known: olean-18-en-3-one (3), ${ }^{3)}$ olean-12-en3 -one (4), ${ }^{3)}$ ferrn-9(11)-ene $(5),{ }^{4}$ ferna-7,9(11)-diene $(6),{ }^{4}$ fern-7-ene (7), ${ }^{4)}$ hop-22(29)-ene (8), ${ }^{4)}$ filic-3-ene (9), ${ }^{4)}$ neohop-12-ene (10), ${ }^{4)}$ adiantoxide (11), ${ }^{1)}$ adiantone (12), ${ }^{5)}$ fern9(11)-en-12-one (13), ${ }^{5}$ 28-hydroxyfern-9(11)-ene (14), ${ }^{1)}$ isoadiantone $(\mathbf{1 5}),{ }^{6}$ isoglaucanone $(\mathbf{1 6}),{ }^{5)}$ hydoxyhopane $(\mathbf{1 7}),{ }^{5)}$ isoadiantol $(\mathbf{1 8})^{5)}$ and hydroxyadiantone (19). ${ }^{5)}$

Compound 1 was obtained as colorless needles, and its high resolution HR-MS showed $\mathrm{M}^{+}$at $\mathrm{m} / z 442.3804$ suggesting the molecular formula to be $\mathrm{C}_{30} \mathrm{H}_{50} \mathrm{O}_{2}$ (Calcd, 442.3810). Its IR spectrum indicated the presence of a hydroxyl and a carbonyl group. The ${ }^{1} \mathrm{H}-\mathrm{NMR}$ spectrum of $\mathbf{1}$ displayed signals for six tertiary and two secondary methyl groups, and the chemical shifts of methyl protons (H-25-H-30) resembled those of filican-3-one (20) (Table 1). The ${ }^{13} \mathrm{C}$ chemical shifts of 1 were also very close to those of 20 (Table 2), except for those of C-2, C-4, C-5, C-6 and C-24. The large down field shifts of C-4 by 22.8 ppm suggested that the hy- droxyl group of 1 was located at C-4.

The heteronuclear multiple bond correlation (HMBC) spectrum of $\mathbf{1}$ also fully corroborated the above observation (Fig. 1). MS of 1 showed distinctive peaks at $m / z 442(\mathrm{M})^{+}$ (100), $427(\mathrm{M}-15)^{+}(10), 424\left(\mathrm{M}-\mathrm{H}_{2} \mathrm{O}\right)^{+}(9), 409(\mathrm{M}-$ $\left.\mathrm{CH}_{3}-\mathrm{H}_{2} \mathrm{O}\right)^{+}(5), 399\left(\mathrm{M}-\mathrm{C}_{3} \mathrm{H}_{7}\right)^{+}(30), 381\left(\mathrm{M}-\mathrm{C}_{3} \mathrm{H}_{7}-\right.$ $\left.\mathrm{H}_{2} \mathrm{O}\right)^{+}$(12), 273 (a) (53), 205 (b) (25), and 191 (c) (27) (Chart 2) which also supported the assigned structure. The orientation of the hydroxyl group was deduced from the nuclear Overhauser enhancement spectroscopy (NOESY) spectrum of 1, which exhibited NOE correlations between the $\mathrm{H}_{3}$ 23 and $\beta$-oriented $\mathrm{H}_{3}-24, \mathrm{H}_{3}-24$ and $\beta$-oriented $\mathrm{H}_{3}-25, \mathrm{H}_{3}-25$ and $\mathrm{H}_{3}-26$, and $\mathrm{H}_{3}-27$ and $\alpha$-oriented $\mathrm{H}_{3}-28$ (Fig. 2), and therefore the $\mathrm{OH}$ group at $\mathrm{C}-4$ must be $\alpha$-oriented. Thus, the structure of 1 was established as $4 \alpha$-hydroxyfilican-3-one.

Compound 2 was obtained as a colorless needles, and its IR spectrum suggested the presence of hydroxyl group in the molecule. Its molecular formula was deduced to be $\mathrm{C}_{30} \mathrm{H}_{50} \mathrm{O}$ by HR-MS ( $\mathrm{M}^{+}$at $\mathrm{m} / \mathrm{z}$ 426.3878; Calcd, 426.3861). The molecular ion peak at $m / z 426(\mathrm{M})^{+}$and the other diagnostic peaks ${ }^{6)}$ at $m / z 411\left(\mathrm{M}-\mathrm{CH}_{3}\right)^{+}(35), 393\left(\mathrm{M}-\mathrm{CH}_{3}-\mathrm{H}_{2} \mathrm{O}\right)^{+}$ (22), 273 (d) (81), $255\left(\mathrm{~d}-\mathrm{H}_{2} \mathrm{O}\right)^{+}(44)$ and 134 (e) (79) (Chart 2) in its MS indicated that 2 is a fernene derivative with a hydroxyl group at ring $\mathrm{A}, \mathrm{B}$ or $\mathrm{C}$ of the molecule. The ${ }^{1} \mathrm{H}-\mathrm{NMR}$ spectrum of $\mathbf{2}$ indicated the presence of six tertiary methyl groups, two secondary methyl groups, one trisubstituted vinylic methine proton and a hydroxyl methine proton (Table 1). The ${ }^{13} \mathrm{C}$-NMR chemical shifts of 2 were similar to those of fern-9(11)-ene (5) ${ }^{6}$ ) except for the signals of C-11, C-12, C-13 and C-18 (Table 2). The large down-field shifts of carbon signals at C-12 by $36.4 \mathrm{ppm}$, and the up-field shift at C18 signal by $c a$. $5.3 \mathrm{ppm}$ suggested that the hydroxyl group of 2 was located at C-12. The HMBC data also fully corrobo-

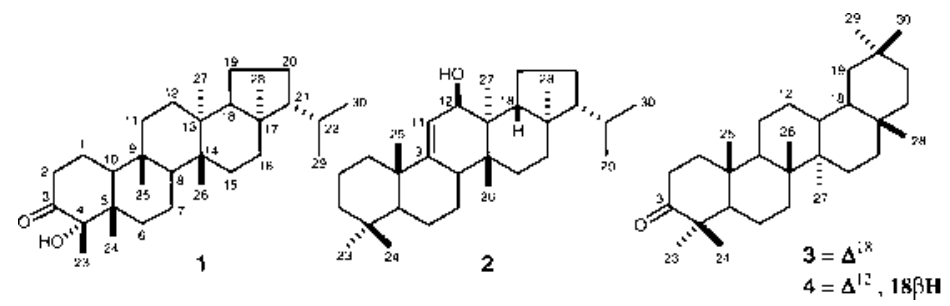

Chart 1 
Table 1. ${ }^{1} \mathrm{H}-\mathrm{NMR}$ Spectral Data $\left(500 \mathrm{MHz}, \mathrm{CDCl}_{3}, \delta\right)$ for $\mathbf{1}-\mathbf{5}$ and $\mathbf{2 0}$

\begin{tabular}{|c|c|c|c|c|c|c|}
\hline${ }^{1} \mathrm{H}$ & 1 & 20 & 2 & 5 & 3 & 4 \\
\hline 23 & 1.154 & $\begin{array}{c}0.900 \\
(\mathrm{~d}, 6.7)\end{array}$ & 0.848 & 0.847 & 1.081 & 1.097 \\
\hline 24 & 0.792 & 0.711 & 0.899 & 0.888 & 1.035 & 1.057 \\
\hline 25 & 0.907 & 0.902 & 1.095 & 1.053 & 0.964 & 1.073 \\
\hline 26 & 0.907 & 0.913 & 0.942 & 0.733 & 1.107 & 1.023 \\
\hline 27 & 1.000 & 0.984 & 0.812 & 0.822 & 0.749 & 0.842 \\
\hline 28 & 0.796 & 0.796 & 0.792 & 0.759 & 1.028 & 0.842 \\
\hline 29 & $\begin{array}{c}0.889 \\
(\mathrm{~d}, 6.7)\end{array}$ & $\begin{array}{c}0.890 \\
(\mathrm{~d}, 6.4)\end{array}$ & $\begin{array}{c}0.907 \\
(\mathrm{~d}, 6.4)\end{array}$ & $\begin{array}{c}0.890 \\
(\mathrm{~d}, 6.4)\end{array}$ & 0.943 & 0.874 \\
\hline 30 & $\begin{array}{c}0.829 \\
(\mathrm{~d}, 6.7)\end{array}$ & $\begin{array}{c}0.829 \\
(\mathrm{~d}, 6.4)\end{array}$ & $\begin{array}{c}0.842 \\
(\mathrm{~d}, 6.4)\end{array}$ & $\begin{array}{c}0.830 \\
(\mathrm{~d}, 6.4)\end{array}$ & 0.943 & 0.874 \\
\hline $2 \alpha$ & $\begin{array}{c}2.970 \\
(\mathrm{ddd}, 2.1,4.9,13.4)\end{array}$ & & & & & \\
\hline $10 \alpha$ & $\begin{array}{c}2.167 \\
(\mathrm{dd}, 3.4,12.8)\end{array}$ & & & & & \\
\hline 11 & & & $\begin{array}{c}5.511 \\
(\mathrm{dd}, 2.4,4.8)\end{array}$ & $\begin{array}{c}5.286 \\
\text { (ddd, } 2.4,2.4,5.1 \text { ) }\end{array}$ & & \\
\hline 12 & & & $\begin{array}{l}3.476 \\
\text { (bs) }\end{array}$ & & & $\begin{array}{c}5.208 \\
(\mathrm{dd} 3.4,3.7)\end{array}$ \\
\hline 19 & & & & & 4.864 & \\
\hline
\end{tabular}

Multiplicity and coupling constant $(J, \mathrm{~Hz})$ are shown in parentheses.

Table 2. ${ }^{13} \mathrm{C}-\mathrm{NMR}$ Spectral Data $\left.{ }^{a}\right)\left(125 \mathrm{MHz}, \mathrm{CDCl}_{3}, \delta\right)$ of $\mathbf{1}-\mathbf{5}$ and $\mathbf{2 0}$

\begin{tabular}{|c|c|c|c|c|c|c|}
\hline & 1 & 20 & 2 & 5 & 3 & 4 \\
\hline 1 & 21.72 & 22.32 & 41.31 & 41.49 & 39.86 & 39.31 \\
\hline 2 & 37.06 & 41.55 & 19.43 & 19.56 & 34.08 & 34.22 \\
\hline 3 & 213.10 & 213.30 & 42.27 & 42.43 & 218.39 & 217.93 \\
\hline 4 & 81.01 & 58.22 & 33.74 & 33.64 & 47.27 & 47.49 \\
\hline 5 & 44.57 & 42.16 & 44.53 & 44.88 & 54.87 & 55.32 \\
\hline 6 & 33.18 & 41.04 & 19.37 & 19.53 & 19.69 & 19.66 \\
\hline 7 & 17.80 & 18.25 & 17.85 & 17.90 & 33.81 & 32.18 \\
\hline 8 & 48.78 & 49.48 & 40.23 & 39.98 & 40.63 & 39.80 \\
\hline 9 & 37.71 & 37.94 & 154.57 & 151.68 & 50.51 & 46.88 \\
\hline 10 & 59.50 & 59.69 & 38.09 & 38.05 & 36.93 & 36.69 \\
\hline 11 & 35.62 & 35.53 & 117.68 & 115.60 & 21.70 & 23.66 \\
\hline 12 & 28.35 & 28.34 & 73.17 & 36.78 & 26.22 & 121.51 \\
\hline 13 & 39.03 & 39.03 & 40.74 & 36.74 & 38.52 & 145.30 \\
\hline 14 & 40.09 & 40.13 & 37.10 & 37.69 & 43.36 & 41.87 \\
\hline 15 & 29.14 & 29.13 & 31.04 & 29.28 & 27.49 & 26.13 \\
\hline 16 & 35.71 & 35.60 & 36.03 & 36.19 & 37.36 & 26.93 \\
\hline 17 & 42.72 & 42.71 & 42.23 & 42.97 & 34.35 & 32.53 \\
\hline 18 & 51.71 & 51.69 & 46.73 & 52.02 & 142.57 & 47.32 \\
\hline 19 & 19.91 & 19.91 & 20.48 & 20.15 & 129.88 & 46.79 \\
\hline 20 & 28.41 & 28.34 & 28.27 & 28.23 & 32.37 & 31.10 \\
\hline 21 & 60.10 & 60.07 & 59.47 & 59.68 & 33.32 & 34.73 \\
\hline 22 & 30.78 & 30.78 & 30.75 & 30.80 & 37.64 & 37.11 \\
\hline 23 & 16.91 & 16.81 & 32.69 & 32.80 & 26.91 & 26.48 \\
\hline 24 & 16.39 & 14.60 & 21.63 & 21.68 & 20.93 & 21.51 \\
\hline 25 & 20.19 & 20.14 & 24.36 & 25.06 & 16.59 & 15.23 \\
\hline 26 & 16.18 & 16.22 & 17.81 & 15.84 & 15.94 & 16.74 \\
\hline 27 & 15.74 & 15.67 & 16.37 & 15.43 & 14.49 & 25.89 \\
\hline 28 & 16.63 & 16.39 & 14.22 & 14.00 & 25.28 & 28.43 \\
\hline 29 & 21.95 & 21.94 & 22.16 & 22.14 & 31.33 & 33.33 \\
\hline 30 & 22.90 & 22.90 & 23.00 & 23.02 & 29.18 & 23.68 \\
\hline
\end{tabular}

a) Assignments were made on the basis of DEPT, ${ }^{1} \mathrm{H}-{ }^{1} \mathrm{H}$ COSY, ${ }^{1} \mathrm{H}-{ }^{13} \mathrm{C}$ COSY and HMBC spectra.

rated the above observation (Fig. 1). The relative stereochemistry at most of the chiral centers of $\mathbf{2}$ as well as the orientation of the $\mathrm{OH}$ group was deduced from its NOESY spectrum which showed NOE interactions as depicted in Fig. 2. The observed interaction of $\mathrm{H}-12(\delta 3.476)$ with $\mathrm{H}_{3}-27$ indicated that the $\mathrm{OH}$ group was $\beta$ oriented. Finally the structure of 2 was confirmed by its preparation from fern-9(11)-en-12-
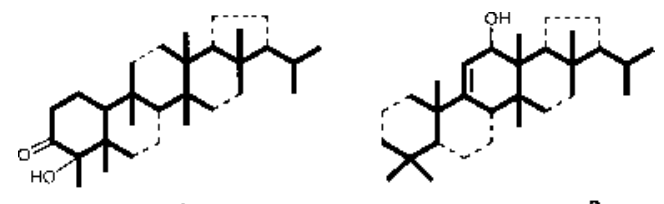

Fig. 1. Partial Structures of $\mathbf{1}$ and $\mathbf{2}$ Derived from HMBC Data

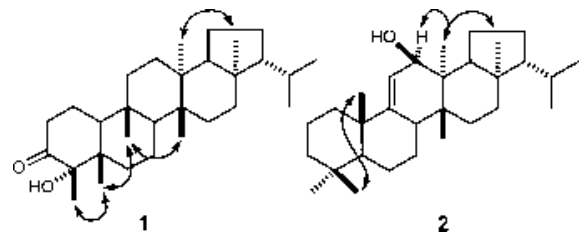

Fig. 2. NOE Interactions Observed in the NOESY Spectra of Compounds 1 and 2

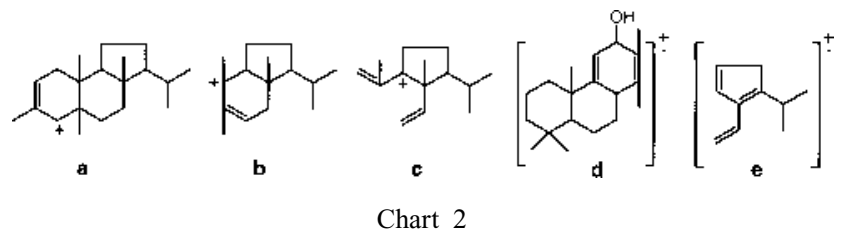

one (21) with $\mathrm{LiAlH}_{4}$. The reaction yielded two products viz. fern-9(11)-en-12 $\alpha$-ol (less polar) (22) and fern-9(11)-en$12 \beta$-ol (more polar) $\left(\mathbf{2}^{\prime}\right)$, of which IR and ${ }^{1} \mathrm{H}-\mathrm{NMR}$ spectra proved to be identical to those of 2 . Thus, the structure of 2 was established as fern-9(11)-en-12 $\beta$-ol.

It was noteworthy that two oleanane compounds $\mathbf{3}$ and $\mathbf{4}$ were obtained from the fronds of Adiantum capillus-veneris (Egyptian origin). Similarity of the Adiantum ferns between Japanese $^{1,2)}$ and Egyptian origin was suggested by detection of many hopane and migrated hopane triterpenoids from both samples. 


\section{Experimental}

General mp.s: uncorr.; EI-MS: $30 \mathrm{eV}$; TLC: on precoated Kiesel gel 60 . ${ }^{1} \mathrm{H}-(500 \mathrm{MHz})$ and ${ }^{13} \mathrm{C}-\mathrm{NMR}(125 \mathrm{MHz})$ spectra: in $\mathrm{CDCl}_{3}$ (TMS as int. standard); HPLC: reverse phase $\mathrm{C}_{18}$ column $(8 \phi \times 250 \mathrm{~mm})$, RI detector, $\mathrm{CH}_{3} \mathrm{CN}-\mathrm{CHCl}_{3}(9: 1)$; CC: silica gel 60 (230- 400 mesh, Merck) and $20 \%$ $\mathrm{AgNO}_{3}$-impregnated silica gel (Mallincrodt); GC: $1.4 \%$ SE-30 on Chromosorb G HP, Oven: $260^{\circ} \mathrm{C}$. (cholestane as int. standard).

The fronds of Adiantum capillus-veneris were collected in Hunan, China and Mansoura, Egypt. Voucher specimens have been deposited in the herbarium of Showa Pharmaceutical University, Tokyo.

Extraction and Separation (Chinese Material) The dried fronds $(50 \mathrm{~g})$ were extracted with hexane three times to give extracts $(1.0 \mathrm{~g})$. The extract was refluxed with benzene for $1 \mathrm{~h}$ and kept for $1 \mathrm{~d}$ at room temperature. The insoluble materials were filtered off (fraction A: fr. A) and the filtrate was evaporated to dryness to afford a gummy residue which was chromatographed over silica gel to give twelve fractions: fr. B (eluted with hexane), fr. C [hexane-benzene $(8: 2)$ ], fr. D, E, F, G [hexane-benzene ( $7: 3)$ ], fr. $\mathrm{H}$ [hexane-benzene $(1: 1)$ ], fr. I, J (benzene), fr. K [benzene-ether $(9: 1)]$, fr. L (ether) and fr. M (methanol). Each eluate was further subjected to silica gel CC and HPLC repeatedly and furnished 1, 2 and the known compounds, viz. 5 (mp $\left.\left.170-171^{\circ} \mathrm{C}, 13 \mathrm{mg}\right),{ }^{4}\right) 6\left(\mathrm{mp} 200-202^{\circ} \mathrm{C}\right.$, $0.5 \mathrm{mg}){ }^{4)} 7\left(\mathrm{mp} 213-214{ }^{\circ} \mathrm{C}, 3 \mathrm{mg}\right),{ }^{4)} \mathbf{8}\left(\mathrm{mp} 211-212^{\circ} \mathrm{C}, 1 \mathrm{mg}\right),{ }^{4)} \mathbf{9}(\mathrm{mp}$ $\left.232-234^{\circ} \mathrm{C}, 0.5 \mathrm{mg}\right),{ }^{4)} \mathbf{1 0}\left(\mathrm{mp} 210-212^{\circ} \mathrm{C}, 0.5 \mathrm{mg}\right){ }^{4)} \mathbf{1 1}(\mathrm{mp} 227-$ $\left.229^{\circ} \mathrm{C}, 47 \mathrm{mg}\right),{ }^{1} \mathbf{1 2}\left(\mathrm{mp} 229-231^{\circ} \mathrm{C}, 90 \mathrm{mg}\right),{ }^{5)} \mathbf{1 3}\left(\mathrm{mp} 229-231^{\circ} \mathrm{C}\right.$, $1 \mathrm{mg}){ }^{5)} \mathbf{1 4}\left(\mathrm{mp} 159-161^{\circ} \mathrm{C}, 4 \mathrm{mg}\right),{ }^{1)} \mathbf{1 5}\left(\mathrm{mp} 236-237^{\circ} \mathrm{C}, 7 \mathrm{mg}\right),{ }^{5)} \mathbf{1 6}(\mathrm{mp}$ $\left.243-245^{\circ} \mathrm{C}, 4 \mathrm{mg}\right),{ }^{5)} \mathbf{1 7}\left(\mathrm{mp} 253-255^{\circ} \mathrm{C}, 1 \mathrm{mg}\right),{ }^{4)} \mathbf{1 8}\left(\mathrm{mp} 213-215^{\circ} \mathrm{C}\right.$, $20 \mathrm{mg})^{5)}$ and 19 (trace) ${ }^{5}$ )

Extraction and Separation (Egyptian Material) The dried fronds $(114 \mathrm{~g})$ were extracted with petroleum ether three times to give the extracts $(3.0 \mathrm{~g})$. The extract was refluxed with benzene for $1 \mathrm{~h}$ and kept for $1 \mathrm{~d}$. The insoluble materials were filtered off (fraction A: fr. A) and the filtrate was evaporated to dryness to afford a gummy residue which was chromatographed over silica gel to give seven fractions: fr. B (eluted with $n$ hexane), fr. $\mathrm{C}[n$ hexane-benzene ( $8: 2)]$, fr. D $[n$ hexane-benzene (7:3)], fr. $\mathrm{E}[n$ hexane-benzene $(1: 1)]$, fr. F $[n$ hexane-benzene $(1: 1)]$, fr. $\mathrm{G}$ (benzene) and fr. $\mathrm{H}$ [benzene- $\left.\mathrm{Et}_{2} \mathrm{O}(9: 1)\right]$. Each eluate was further subjected to silica gel CC and HPLC repeatedly and furnished 3, 4 and the known compounds, viz. 11 (mp $\left.229-230^{\circ} \mathrm{C}, 158 \mathrm{mg}\right),{ }^{1)} 12$ (mp $227-230{ }^{\circ} \mathrm{C}$, $145 \mathrm{mg}),{ }^{5)} \mathbf{1 5}\left(\mathrm{mp} 236-237^{\circ} \mathrm{C}, 19 \mathrm{mg}\right),{ }^{5)} \mathbf{1 6}\left(\mathrm{mp} 243-245^{\circ} \mathrm{C}, 8 \mathrm{mg}\right)^{5)}$ and 18 (mp $\left.\left.213-215^{\circ} \mathrm{C}, 30 \mathrm{mg}\right) .^{5}\right)$

$4 \alpha$-Hydroxyfilican-3-one (1): Fraction $\mathrm{K}$ was chromatographed over silica gel with benzene followed by crystallization from $\mathrm{MeOH}-\mathrm{CHCl}_{3}$ to give $\mathbf{1}$ (3 mg). mp $263-265^{\circ} \mathrm{C} .[\alpha]_{\mathrm{D}}-4.8^{\circ}\left(c=0.2, \mathrm{CHCl}_{3}\right) . v_{\max }^{\mathrm{KBr}} \mathrm{cm}^{-1}, 3505$ $(\mathrm{OH})$ and $1719(\mathrm{C}=\mathrm{O})$. EI-MS $\mathrm{m} / z$ (rel. int. \%): $442(\mathrm{M})^{+}(100), 427$
$\left(\mathrm{M}-\mathrm{CH}_{3}\right)^{+}(10), 424\left(\mathrm{M}-\mathrm{H}_{2} \mathrm{O}\right)^{+}(9), 409\left(\mathrm{M}-\mathrm{H}_{2} \mathrm{O}-\mathrm{COCH}_{3}\right)^{+}(5), 399$ (30), 381 (12). HR-MS; $\mathrm{M}^{+} \mathrm{m} / z$ : 442.3804 (Calcd for $\mathrm{C}_{30} \mathrm{H}_{50} \mathrm{O}_{2}: 442.3801$ ). ${ }^{1} \mathrm{H}$ - and ${ }^{13} \mathrm{C}-\mathrm{NMR}$ spectral analyses: Tables 1 and 2.

Fern-9(11)-12 $\beta$-ol (2): Fraction F was chromatographed over silica gel with $n$ hexane-benzene $(7: 3)$ and then each of the fractions was subjected to preparative HPLC followed by crystallization to give 2 (3 mg). $\mathrm{mp} 191-$ $192^{\circ} \mathrm{C} .[\alpha]_{\mathrm{D}}-47.4^{\circ}\left(c=0.2, \mathrm{CHCl}_{3}\right) . v_{\max }^{\mathrm{KBr}} \mathrm{cm}^{-1}: 3450(\mathrm{OH})$ and 1018 (C-O). EI-MS $m / z$ (rel. int. \%): $426(\mathrm{M})^{+}(15), 441\left(\mathrm{M}-\mathrm{CH}_{3}\right)^{+}(35), 393$ $\left(\mathrm{M}-\mathrm{H}_{2} \mathrm{O}-\mathrm{CH}_{3}\right)^{+}$(22), 273 (81), 255 (44), 134 (79). HR-MS; $\mathrm{M}^{+} m / z$ : 426.3878 (Calcd for $\mathrm{C}_{30} \mathrm{H}_{50} \mathrm{O}_{2}: 426.3861$ ). ${ }^{1} \mathrm{H}$ - and ${ }^{13} \mathrm{C}-\mathrm{NMR}$ spectral analyses: Tables 1 and 2.

Olean-18-en-3-one (3): $\mathrm{mp} 183-185.5^{\circ} \mathrm{C} . v_{\max }^{\mathrm{KBr}} \mathrm{cm}^{-1} ; 1700(\mathrm{C}=\mathrm{O})$. EIMS $m / z$ (rel. int. \%): $424(\mathrm{M})^{+}(46), 409\left(\mathrm{M}-\mathrm{CH}_{3}\right)^{+}(68), 218(11), 205$ (59), 204 (73), 177 (100). ${ }^{1} \mathrm{H}-\mathrm{NMR} \delta: 1.081(3 \mathrm{H}, \mathrm{s}, \mathrm{H}-23), 1.035$ (3H, s, H24), 0.964 (3H, s, H-25), 1.107 (3H, s, H-26), 0.749 (3H, s, H-27), 1.028 (3H, s, H-28), 0.943 (3H, s, H-29), $0.943(3 \mathrm{H}, \mathrm{s}, \mathrm{H}-30), 4.864(1 \mathrm{H}, \mathrm{s}, \mathrm{H}-$ 19).

Olean-12-en-3-one (4): $\mathrm{mp} 166-167^{\circ} \mathrm{C}, v_{\max }^{\mathrm{KBr}} \mathrm{cm}^{-1} ; 1700(\mathrm{C}=\mathrm{O})$. EIMS $m / z$ (rel. int. \%): $424(\mathrm{M})^{+}(26), 409\left(\mathrm{M}-\mathrm{CH}_{3}\right)^{+}(19), 218(100), 205$ (23), 203 (63), 189 (73). ${ }^{1} \mathrm{H}-\mathrm{NMR} \delta$ : 1.097 (3H, s, H-23), 1.057 (3H, s, H24), 1.073 (3H, s, H-25), 1.023 (3H, s, H-26), 1.042 (3H, s, H-27), 0.842 $(3 \mathrm{H}, \mathrm{s}, \mathrm{H}-28), 0.874(3 \mathrm{H}, \mathrm{s}, \mathrm{H}-29), 0.874(3 \mathrm{H}, \mathrm{s}, \mathrm{H}-30), 5.208(1 \mathrm{H}, \mathrm{dd}$, $J=3.4,3.7 \mathrm{~Hz}, \mathrm{H}-12) .{ }^{3)}$

LiAlH 4 Reduction of $2121(20 \mathrm{mg})$ was reduced with $\mathrm{LiAlH}_{4}$ in anhyd. ether and treated in the usual manner. The reaction product was purified by silica CC to give $\mathbf{2}^{\prime}(6 \mathrm{mg})$ and $\mathbf{2 2}(4 \mathrm{mg})$.

Acknowledgements The authors are grateful to Mr. Y. Takase for mass measurements.

\section{References}

1) Nakane T., Arai Y., Masuda K., Ishizaki Y., Ageta H., Shiojima K., Chem. Pharm. Bull., 47, 543-547 (1999).

2) Shiojima K., Arai Y., Nakane T., Ageta H., Cai S.-Q., Chem. Pharm. Bull., 45, 1608-1610 (1997).

3) Shiojima K., Masuda K., Suzuki H., Lin T., Ooishi Y., Ageta H., Chem. Pharm. Bull., 43, 1634-1639 (1995).

4) Ageta H., Shiojima K., Arai Y., Suzuki H., Kiyotani T., Chem. Pharm. Bull., 42, 39-44 (1994).

5) Shiojima K., Sasaki Y., Ageta H., Chem. Pharm. Bull., 41, 268-271 (1993).

6) Shiojima K., Arai Y., Masuda K., Takase Y., Ageta T., Ageta H., Chem. Pharm. Bull., 40, 1683-1690 (1992). 